\title{
Incidence of Quantum Confinement on Dark Triplet Excitons in Carbon Nanotubes
}

\author{
J. Palotás, M. Negyedi, S. Kollarics, A. Bojtor, P. Rohringer, T. Pichler, and F. Simon*
}

Cite This: ACS Nano 2020, 14, 11254-11261

Read Online

ACCESS | Lلll Metrics \& More | 回 Article Recommendations | S1 Supporting Information

ABSTRACT: The photophysics of single-wall carbon nanotubes (SWCNTs) is intensively studied due to their potential application in light harvesting and optoelectronics. Excited states of SWCNTs form strongly bound electron-hole pairs, excitons, of which only singlet excitons participate in application relevant optical transitions. Long-living spin-triplet states hinder applications, but they emerge as candidates for quantum information storage. Therefore, knowledge of the triplet exciton energy structure, in particular in a SWCNT chirality dependent manner, is greatly desired. We report the observation of light emission from triplet state recombination, i.e., phosphorescence, for several SWCNT chiralities using a purpose-built spectrometer. This yields the singlet-triplet gap as a function of the SWCNT diameter, and it follows predictions based on quantum confinement effects. Saturation under high microwave power (up to $10 \mathrm{~W}$ ) irradiation

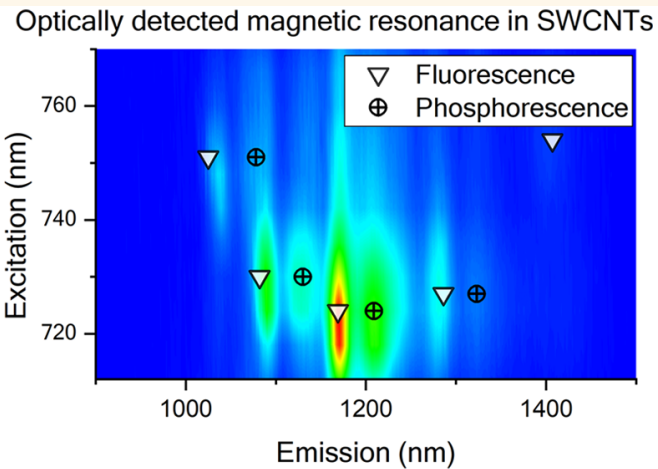
allows the spin-relaxation time for triplet states to be determined. Our study sensitively discriminates whether the lowest optically active state is populated from an excited state on the same nanotube or through Förster exciton energy transfer from a neighboring nanotube.

KEYWORDS: carbon nanotubes, optically detected magnetic resonance, relaxation times, quantum confinement, molecular rulers, Föster exciton transfer

U nderstanding the photophysics of novel materials is crucial for their effective application in both light harvesting and light generation, which are relevant for diverse fields including energy applications, optoelectronics, telecommunications, photonics, and quantum technology. The fundamental processes involve either the generation or the recombination of an electron-hole pair. This pair is often correlated due to Coulomb interaction, and it makes a quasiparticle known as an exciton. ${ }^{1}$ Typical binding energies are a few millielectronvolts in semiconductors, ${ }^{2}$ but they can be as large as a few $100 \mathrm{meV}$ in single-wall carbon nanotubes (SWCNTs) as the Coulomb interaction is only weakly screened in the one-dimensional surrounding. ${ }^{3,4}$ Only excitons with a singlet spin wave function (or a singlet exciton) can be generated in the absorption of a single photon, ${ }^{5,6}$ but the nonspin-conserving spin-orbit coupling can generate an exciton in a spin-triplet state (a triplet-exciton). This low probability process is known as intersystem-crossing (ISC). ${ }^{7}$ The ISC results in the accumulation of triplet states, the triplet-exciton decay being a slow process as it again violates spin conservation.

Knowledge of the singlet/triplet energy structure as well as the ISC and the triplet decay dynamics is of great importance for photophysics applications. Among others, the presence of triplet states hinders light harvesting due to the long recovery of the photoactive unit once it enters a triplet state. Triplet excited states also pose a challenge for light generation: an injected electron-hole pair forms a triplet state with $75 \%$ probability; thus, the theoretical limit on the internal quantum efficiency for a fluorescence based light emitting device is $25 \%$ (ref 8). On the other hand, the ability to address and manipulate a long-living triplet state can be exploited for quantum information storage such as in the well-known NV center in diamond. ${ }^{9,10}$ An additional motivation arises from the possible use of excitons in switching electronics. ${ }^{11,12}$

The photophysics of SWCNTs have been intensively studied due to their potential for light related applications, and the basic optical properties are well-known. ${ }^{5,13,14}$ Light emission in SWCNTs arises from a bright, singlet exciton level, or $S_{1}$, which is one of four states due to the twofold, $\mathrm{K}-\mathrm{K}^{\prime}$ degeneracy of the conduction and valence bands. ${ }^{3,415}$ The

Received: April 14, 2020

Accepted: August 13, 2020

Published: August 13, 2020

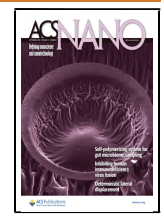


a)
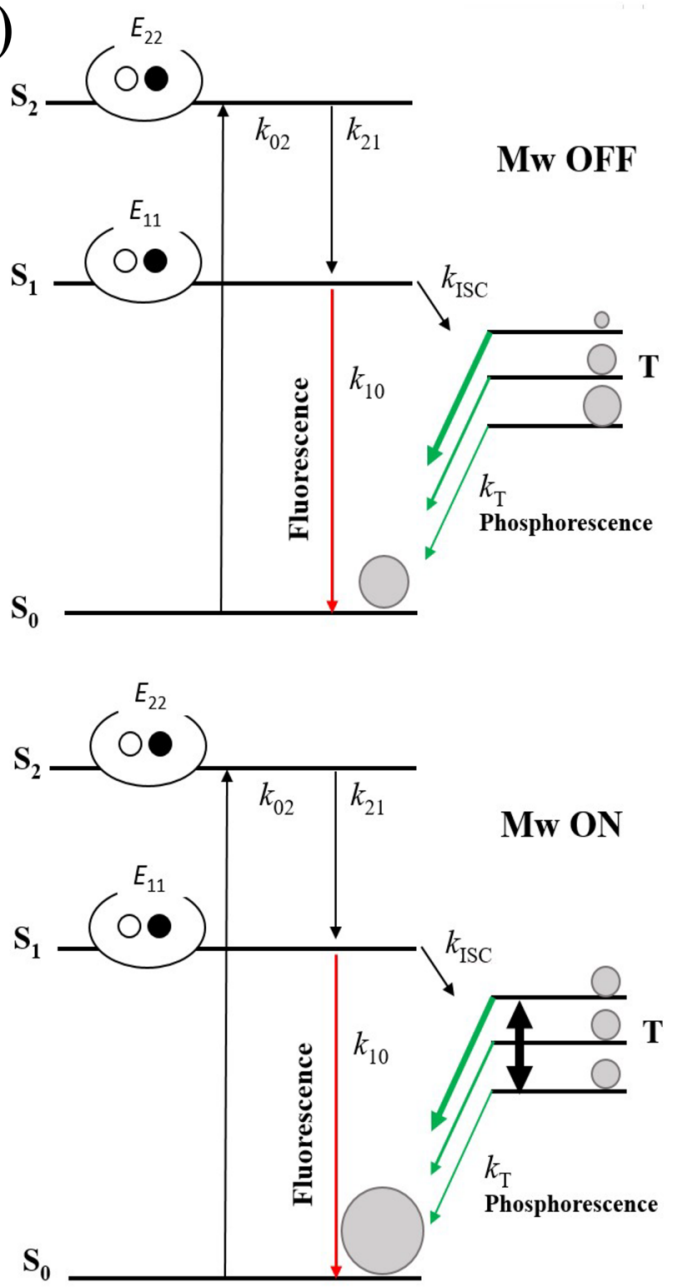

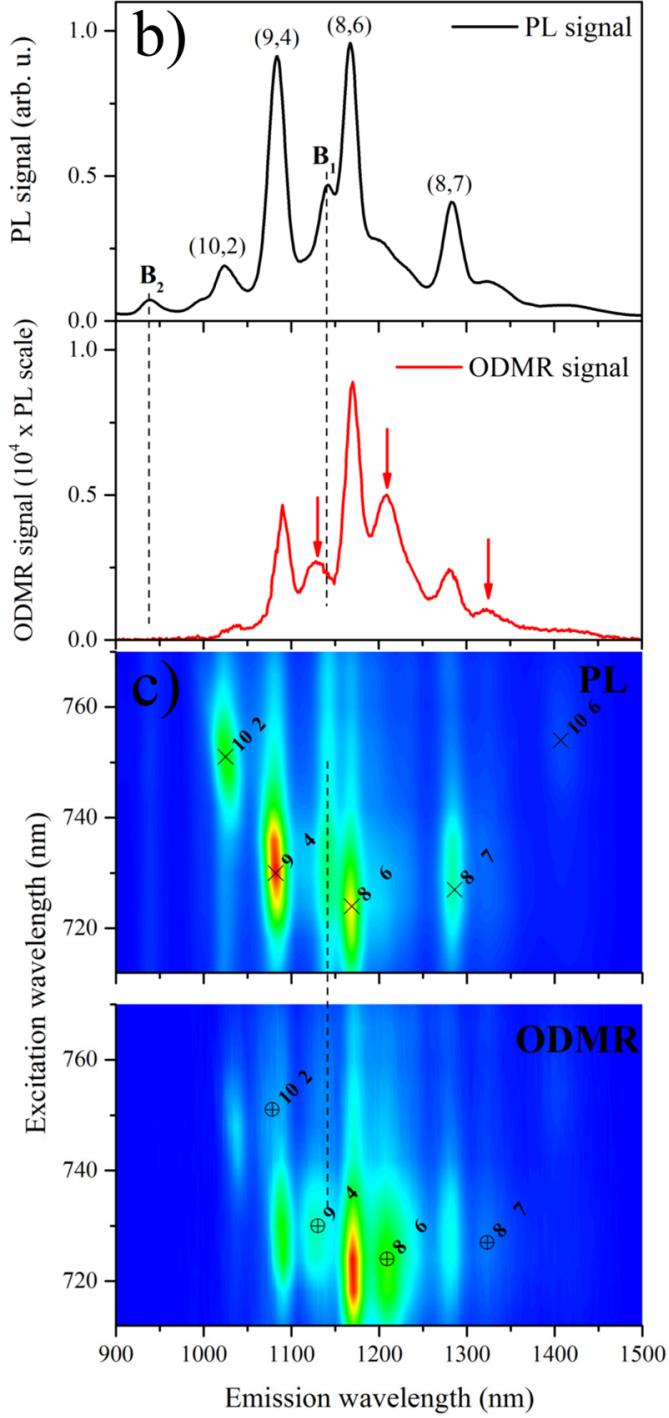

Figure 1. Jablonski diagram and ODMR results on SWCNTs. a, The Jablonski diagram for the SWCNTs which illustrates the ODMR induced processes for fluorescence and phosphorescence. b. Individual PL and ODMR spectra when excited at $724 \mathrm{~nm}$. Chiral indices are given, vertical arrows indicate the phosphorescent sidebands, and vertical dashed lines highlight "bundle-peaks" (B1 and B2). c, PL and ODMR maps of SWCNTs. Maxima of the PL signals are indicated by $\times$, the corresponding $(n, m)$ chiral indices are also given, and $\oplus$ indicates the maxima of the phosphorescence sidebands on the ODMR map. Note the absence of the "bundle-peak" (vertical dashed lines) on the ODMR map.

other three exciton levels are referred to as dark since light emission from these levels is not allowed in first order by momentum and angular momentum conservation. ${ }^{3-6}$ The lowest-lying exciton level is dark, separated by a few millielectronvolts from the bright exciton level. ${ }^{15}$ This causes the nonradiative recombination processes to dominate and the radiative fluorescent process to have a low probability with a quantum yield below $1 \%$ (refs 16 and 17). The $S_{1}$ bright exciton state is usually populated by irradiating the second bright singlet exciton level, $S_{2}$, which rapidly relaxes to $S_{1}$ by internal conversion. Given that both the absorption and emission energies depend strongly on the SWCNT $(n, m)$ chiral indices, the fluorescent studies have a great analytic value to characterize, e.g., the abundance of a particular SWCNT in a sample. $^{14}$

In addition to the 4 spin-singlet exciton states, each can form a spin-triplet state (referred to as triplet in the following), i.e., altogether the SWCNTs have 16 exciton levels of which only one is bright, that gives rise to the usual band gap fluorescence. ${ }^{5,6}$ Recent magneto-photoluminescence showed the influence of the triplet states on the photoluminescence emission. ${ }^{18}$ The triplet levels lie below the singlet, due to the exchange interaction, which mimics Hund's rule for atoms.

Here, we report the direct observation of the phosphorescence signal from the triplet levels in pristine SWCNTs in an $(n, m)$ chiral index resolved manner. The key to this was the recent development of a unique ODMR spectrometer ${ }^{19}$ which operates with a tunable laser source, thus selecting a particular absorption energy, and by analyzing the wavelength of the emitted signal under magnetic resonance conditions. Another challenge to overcome was the near-infrared emission range for most SWCNTs where the detector efficiency is lower. Our experiment yields the singlet-triplet energy gap for several $(n$, $m$ ) chiralities, and the result is in good agreement with the theoretically predicted values in ref 20 , which indicates that the exchange energy is strongly diameter dependent. Intensive 
microwave irradiation allowed the spin-relaxation time to be determined for the triplet levels. We observe an absence of the so-called bundle peaks in the ODMR spectra, which indicates a dominance of Förster exciton energy transfer over conventional optical decay routes for neighboring nanotubes.

\section{RESULTS AND DISCUSSION}

Figure 1a shows the Jablonski diagram of the SWCNT photophysics for the lowest-lying bright exciton. ${ }^{21-24}$ Note that excited exciton states with $L \neq 0$ form a Rydberg series, and these cannot be excited in single photon experiments. ${ }^{25}$ The figure illustrates that the $S_{2}$ bright singlet exciton level is excited from the ground state by absorption, which corresponds to an optical transition between the second van Hove singularity pair ${ }^{13,14}$ in the single-particle description. This excited state relaxes to the $S_{1}$ bright exciton state by internal conversion. Its recombination to the ground state yields the fluorescence, but it can also crossover to the triplet state, $\mathrm{T}$, due to spin-orbit interaction. The recombination of the triplet is not allowed in the first order by spin conservation, but again spin-orbit coupling can give rise to a faint phosphorescent signal. The detection of the triplet emission using magnetic resonance is called optically detected magnetic resonance (ODMR, refs 21-23), which is also used herein.

Understanding the triplet energy structure, the singlettriplet energy gap, $E_{\mathrm{ST}}$, is important for future optoelectronics applications of SWCNTs ${ }^{26,27}$ or to assess the utility of the triplet level, e.g., quantum information storage potential. ${ }^{28,29}$ In particular, it would be important to gain this information in an $(n, m)$ specific manner as a typical nanotube sample contains a large number of various SWCNTs. Spectroscopic evidence for the existence of triplet levels came from chemically modified SWCNTs with an enhanced phosphorescent activity, ${ }^{30}$ more recently from ODMR studies, ${ }^{31}$ and also from magnetophotoluminescence. ${ }^{18}$

The $E_{\mathrm{ST}}$ for the chemically modified SWCNTs was found to be about twice as large as the theoretically predicted value. ${ }^{20}$ The ODMR study did not resolve the separate fluorescence and phosphorescence; i.e., the direct observation of light emission from the triplet state in pristine SWCNTs remains elusive.

In Figure $1 \mathrm{~b}$, we show individual PL and ODMR spectra at $77 \mathrm{~K}$, when excited at $724 \mathrm{~nm}$. The ODMR spectrum is the change in the optical signal due to the microwave irradiation, $\Delta \mathrm{PL}$. Note that the PL spectrum is 4 orders of magnitude larger than the ODMR signal. The ODMR signal emerges as the $S_{1}$ singlet exciton state undergoes intersystem crossing to the triplet, $\mathrm{T}$, state due to spin-orbit coupling. Recombination of the three triplet levels to the ground state can give rise to a faint phosphorescent signal due to the low probability as a result of spin-conservation. Again, spin-orbit coupling enables recombination of the triplet states. The weakness of this process hinders its direct observation in optical studies, and these states are often referred to as dark triplet excitons. It was shown in ref 30 that hydrogen coverage of SWCNTs can enhance a sideband signal which was associated with the phosphorescence of SWCNTs. The master equation for the triplet population, $[\mathrm{T}]$ reads:

$$
\frac{\mathrm{d}[\mathrm{T}]}{\mathrm{d} t}=k_{\mathrm{ISC}}\left[\mathrm{S}_{1}\right]-k_{\mathrm{T}}[\mathrm{T}]
$$

where $\left[\mathrm{S}_{1}\right]$ is the population of the excited state. Under steady state conditions, eq 1 leads to a finite triplet population, with the photoactive states being effectively "trapped" in the longliving triplet state. This means that ISC not only gives rise to a finite triplet population but it also reduces the fluorescent signal.

In principle, the ISC process can be reversible due to triplet-triplet interactions at high irradiation fluences ${ }^{31}$ or parity flipping due to adsorbed molecules. ${ }^{32}$ In our case, neither of these mechanisms can be significant, and our wavelength resolved approach enables discrimination for origin of the emitted photon, as shown below.

Figure 1a illustrates the origin of the ODMR signal as follows. The three sublevels of the triplet are split either by zero-field splitting (ZFS) or by an external magnetic field (the Zeeman effect). The presence of a strong ZFS is typical for strongly localized (Frenkel) excitons; ${ }^{22}$ however, when ZFS is small, which is the case for a larger Mott-Wannier type exciton such as in SWCNTs, ${ }^{33}$ an external magnetic field can split the levels. The population of the three sublevels is usually unequal immediately after ISC as they couple differently to the lattice due to their differing orbital arrangement. Similarly, the decay rates to the ground states of the individual triplet sublevels are also usually different. An intensive microwave irradiation on the magnetic resonance condition equilibrates the populations of the triplet sublevels, thereby affecting the phosphorescent signal. A consideration of the number of available states ${ }^{22}$ yields (also discussed in the Supporting Information) that the microwave induced additional phosphorescent flux equals the additional fluorescent flux; i.e., the more states are liberated from the triplet state, the more fluorescent transitions can occur. We note that our signal is positive; i.e., the presence of microwave irradiation increases both the fluorescent and the phosphorescent signals. A previous ODMR report in SWCNTs did not resolve these two processes as no wavelength analysis of the emitted light was performed. ${ }^{31}$

For SWCNTs, both the $S_{0} \rightarrow S_{2}$ absorption and the $S_{1} \rightarrow S_{0}$ fluorescence emission depend on the $(n, m)$ indices, ${ }^{13}$ which allowed a chiral index assignment of the individual fluorescence peaks. ${ }^{14}$ When plotted as a function of excitation and emission, the individual PL spectra yields a PL map, which is shown in Figure 1c. The individual ODMR spectra can be combined in a similar manner, which yields the ODMR map in Figure 1c. The resolution is $1 \mathrm{~nm}$ for the emission and $6 \mathrm{~nm}$ for the excitation. The PL map ${ }^{13}$ contains well-known features, including some strong emission peaks at the corresponding excitation energy, which are indicated with " $X$ " in the figure, and the corresponding $(n, m)$ chiral indices are also given.

The emission and excitation energies in our PL map (values are given in the Supporting Information) slightly differ from well-known literature values, ${ }^{34}$ due to a differing surfactant, temperature, or level of bundling. However, SWCNT chiralities could be unambiguously identified due to the patterned nature of the transition energies. ${ }^{13,14}$ In addition, the PL map also contains features which are colloquially known as "bundle-peaks"33,35,36 and are indicated by vertical solid lines. These features appear when a few nanotubes form a small and interacting aggregate structure and an SWCNT with appropriate transition energy absorbs light. The resulting exciton is then transferred to another SWCNT in the bundle, which then emits at its characteristic emission wavelength. ${ }^{35,36}$ This process leads to a characteristic feature of the bundle peaks that they appear as vertical lines in the PL maps. These peaks are absent in the ODMR map, which is discussed further below. 
We note that the main fluorescent peaks do not appear at exactly the same position on the PL and ODMR studies beyond the experiment accuracy as we discuss in the Supporting Information. We believe that this effect is due to the known inhomogeneous broadening of the PL lines ${ }^{37}$ either due to a different charging, defects, or a slightly different environment. We speculate that the ISC transition rates and thus the triplet population is affected by these factors; i.e., the microwave induced fluorescent peaks in the conventional PL studies should not necessarily match those in the ODMR results. However, this effect requires further study.

We provide additional excitation and emission profiles in the Supporting Information to support the above claim that indeed all major peaks have a longer wavelength satellite. An exception is the $(10,6)$ peak which should show a satellite in the wavelength region where our spectrometer sensitivity abruptly drops. It is interesting to note that the phosphorescent peaks have the same integrated intensity as the fluorescent ones but are broader. The same intensity is explained above, and the larger line width could be related to a larger sensitivity of the triplet level to the above-mentioned environmental factors. We note that the Zeeman splitting of the triplet levels $(9 \mathrm{GHz})$ is much smaller than the extra broadening ( 1 at $1100 \mathrm{~nm}$ wavelength corresponds to $250 \mathrm{GHz}$ ).

The observation of the phosphorescence in ODMR for four SWCNT chiralities allows the chirality resolved singlet-triplet energy gap, $\Delta_{\mathrm{ST}}$, to be determined. The result is shown in Figure 2 together with the theoretical prediction by Capaz et

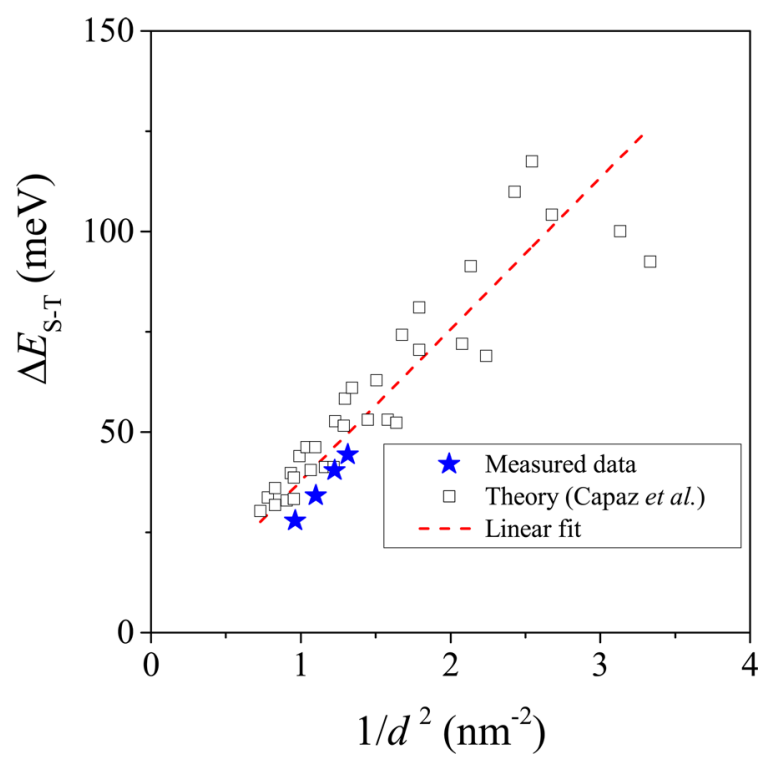

Figure 2. Singlet-triplet gap in SWCNTs. Magnitude of the singlet-triplet energy gap for the four SWCNTs which were identified in our study $(\star)$ as a function of the inverse of the squared nanotube diameter, $1 / d^{2}$. The theoretical prediction by Capaz et al. (ref.20) is shown with $\square$ symbols along with a linear fit to the data.

al. (ref 20). The latter calculation was based on considering the variation of the exchange interaction due to the finite size quantum confinement.

The exciton size scales with the tube diameter, $d$, as it is the only length scale in the problem. The exciton binding energy scales with $1 / d$ as the Coulomb interaction energy scales with the inverse distance. The singlet-triplet splitting is due to the exchange energy, and the latter varies as $1 / d^{2}$ as it is due to the electric dipole self-interaction of a neutral charge distribution. ${ }^{38}$ A similar, $1 / d^{2}$ dependence was observed for the energy difference between the bright and the defect-brightened, otherwise dark, excitons. ${ }^{39}$ This is due to the fact that the bright-dark exciton splitting is also related to the exchange interaction according to ref 38 .

Our experimental data matches well with the theoretical prediction which indicates that the theoretical model was indeed appropriate. This agreement also enables the future identification of phosphorescence in SWCNTs with chiralities which are not presented herein. Certainly, extending our measurements toward SWCNTs with largely different diameters (such as, e.g., the (6,4) and $(6,5)$ SWCNTs) would improve the confidence in the agreement.

We studied the microwave power, $p$, dependence of a selected peak (of the $(8,6)$ SWCNT) at $1169 \mathrm{~nm}$ emission and $724 \mathrm{~nm}$ excitation in the ODMR map, in order to gain information about the characteristic spin-dynamics time scales: $T_{1}$ (spin-lattice relaxation time) and $T_{2}$ (spin-spin relaxation time). The magnitude of these parameters determines whether the SWCNT triplet states could be used, e.g., for quantum information storage purposes.

The power dependence of the ODMR signal is shown in Figure 3. The ODMR signal is expected to follow the

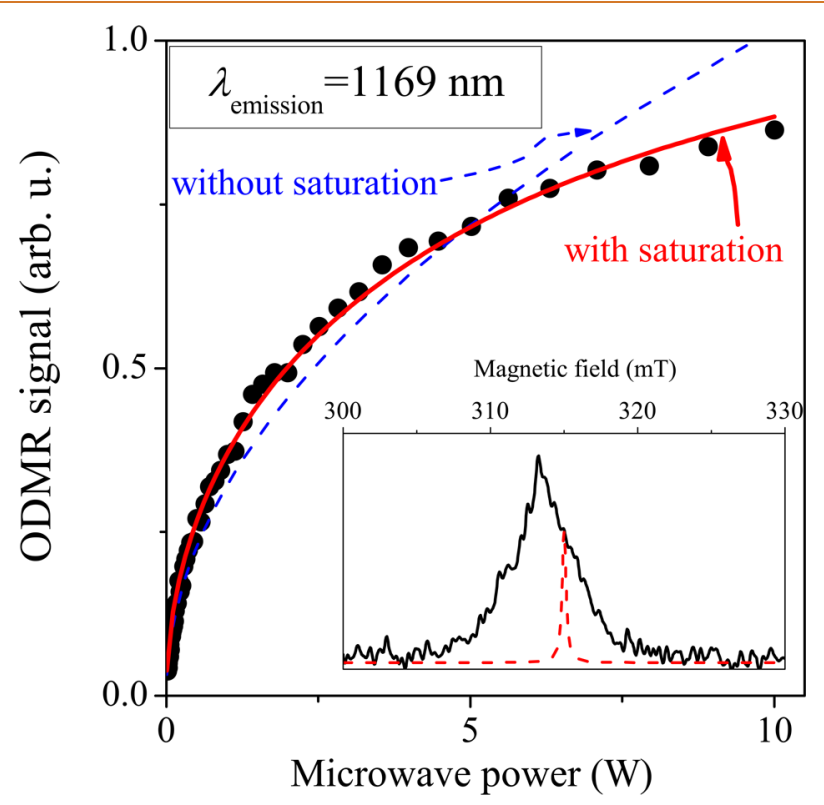

Figure 3. Microwave power dependence of the ODMR signal. The emission wavelength is $1169 \mathrm{~nm}$ that corresponds to the $(8,6)$ SWCNT. Dashed and solid curves show a fit without and with saturation effects included, respectively. The inset shows a magnetic field swept ODMR spectrum, and the signal of an individual spin-packet is also indicated with a dashed line.

population change in the triplet sublevels, induced by the microwave excitation. For low microwave powers, it follows the strength of the microwave magnetic field, ${ }^{40} B_{1}$, ODMR $\propto B_{1} \propto \sqrt{p}$; however, at sufficiently high powers, a saturation could be observed as ${ }^{40}$

$$
\text { ODMR } \propto \sqrt{\frac{p}{1+C p Q \gamma^{2} T_{1} T_{2}}}
$$


a)

\begin{tabular}{|c|c|}
\hline Donor: $(9,4)$ & Acceptor $(7,6)$ \\
\hline
\end{tabular}

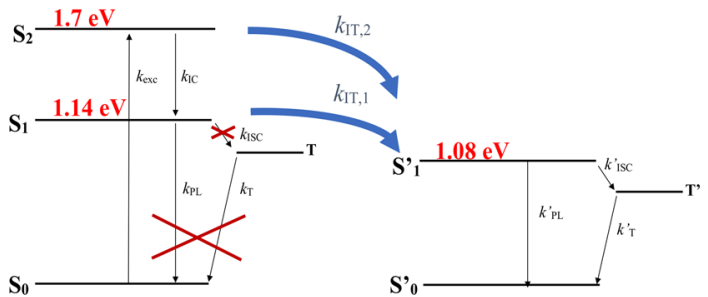

b)

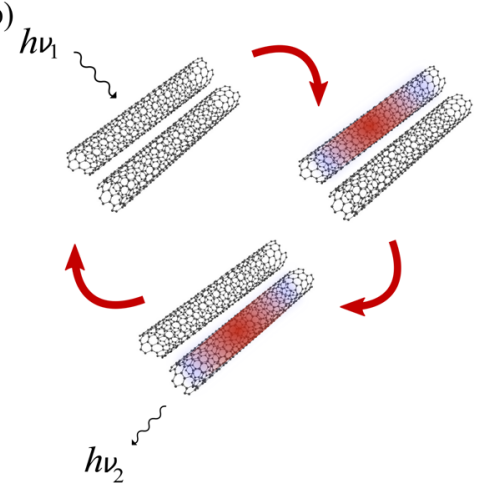

Figure 4. Jablonski diagram (a) and schematics (b) for two neighboring SWCNTs. The exciton created on the donor SWCNT (the $(9,4)$ in this case) is transferred to the neighboring acceptor tube (the $(7,6)$ tube in this case) with a high probability (thick arrows); thus, the usual relaxation processes (crossed-out arrows) do not occur. Schematic depiction of the dominating exciton energy transfer process: the absorbed light forms an exciton which is then transferred to the acceptor SWCNT where the exciton recombination and light emission occurs.

Herein, $Q=1000$ is the quality factor of our microwave cavity, and $C=2.2 \times 10^{-12} \mathrm{~T}^{2} / \mathrm{W}^{2}$ is a resonator constant which links the generated microwave magnetic field strength to the power and is resonator mode dependent ${ }^{41}$ (it is the cylindrical TE011 mode in our case). Besides the gyromagnetic ratio, $\gamma / 2 \pi=28$ $\mathrm{GHz} / \mathrm{T}$, the formula allows the $T_{1} T_{2}$ product $^{42}$ to be obtained when saturation is present.

Figure 3 shows that a fit to the power dependent ODMR signal without saturation does not account for the data at high microwave powers. When saturation is included, eq 2 describes well the data in the whole power range, and we obtained $\sqrt{T_{1} T_{2}}=36(1) \mathrm{ns}$. Although, the data itself does not allow the values of $T_{1}$ and $T_{2}$ to be disentangled separately, we argue below that most probably $T_{2}=T_{1}$ holds due to the short value of $T_{1}$. This case can be related to a homogeneous line width of $\Delta B_{\text {hom }}=1 / \gamma T_{1}=0.16 \mathrm{mT}$.

In general the $T_{2}<T_{1}$ hierarchy holds, except when $T_{1}$ becomes so short that it shortens the $T_{2}$ as well, ${ }^{43}$ which results in $T_{2}=T_{1}$. For most materials, $T_{2}$, and the related line width $\Delta B_{\text {dipole }}=1 / \gamma T_{2}$ is due to the dipole-dipole interaction between like spins, which can be quantified using the van Vleck formula. ${ }^{44}$ A significant exciton-phonon interaction ${ }^{45}$ could also lead to additional dephasing and thus a shorter value of $T_{2}$. However, it is expected that, at low temperatures, this effect vanishes and the dipole-dipole interaction dominates. A reasonable estimate (calculation is given in the Supporting Information) for the maximum value of $\Delta B_{\text {dipole }}=0.02 \mathrm{mT}$ can be obtained for a triplet exciton to triplet exciton distance of 10 $\mathrm{nm}$, which is close to the exciton Bohr radius; thus, it can be regarded as a lower limit for their separation. ${ }^{6}$ However, this value of $\Delta B_{\text {dipole }}$ is smaller than the above $\Delta B_{\text {hom }}$ by an order of magnitude. This means that dipole broadening cannot account for the observed $T_{1} T_{2}$ value, and we are thus led to conclude that $T_{1}=T_{2}=36(1) \mathrm{ns}$ at $77 \mathrm{~K}$ in the SWCNTs.

We note that the spectral line width of a magnetic field swept ODMR spectrum is about $5 \mathrm{mT}$ (see the inset of Figure $3)$, which is much larger than the above-mentioned homogeneous line width. Portis showed ${ }^{42}$ that it is the line width of the so-called spin-packets with line width $1 / \gamma T_{2}$ (shown with a dashed curve in the inset of Figure 3) that enters the saturation formula and that the visible line width (or signal envelope) does not affect it. As per the above short values of $T_{1}$ and $T_{2}$, we believe that it is caused by the electron-phonon interaction, which is mediated to the spin state through spin-orbit coupling, ${ }^{43}$ but its origin requires additional theoretical studies. This short value of $T_{1,2}$ appears to be prohibitively short if the triplet state would be used to store quantum information. Although SWCNTs appear particularly advantageous for such purposes, ${ }^{29}$ given the compatibility of the emission and absorption wavelengths with the telecommunication windows $(\approx 1300-1550 \mathrm{~nm})$, a $T_{1}$ of a few milliseconds or microseconds is required for a meaningful operation. ${ }^{29,46}$

We mentioned above that the "bundle-peaks", $B_{1}$ and $B_{2}$, are absent in the ODMR spectra as Figure 1c demonstrates. As mentioned, these are the emission peaks which are related to a Förster exciton energy transfer process between two neighboring nanotubes which form a small bundle. This absence in the ODMR cannot be due to experimental factors: the $B_{2}$ peak falls well within the range of sensitivity of our system, and this spectral range is free of other SWCNT peaks; still the ODMR spectrum shows no trace of a peak. Concerning the $\mathrm{B}_{1}$ peak, the spectral range has more peaks; however, the PL map shows that this bundle peak is particularly strong and has a comparable intensity to the $(9,4)$ and $(8,6)$ peaks. However, the ODMR spectrum contains no signature of these peaks.

The relevant Jablonski diagram for the $\mathrm{B}_{1}$ line in Figure $1 \mathrm{c}$ is shown in Figure 4. Here, the donor SWCNT is the $(9,4)$, and the exciton is transferred to the acceptor $(7,6)$ which then radiates the observed emission (thick curved arrows in the figure). It cannot be decided from the present data whether the exciton energy transfer occurs from the $S_{2}$ (intertube transfer process, $k_{\mathrm{IT}, 1}$ ) or $S_{1}$ (intertube transfer process: $k_{\mathrm{IT}, 2}$ ) levels of the $(9,4)$ donor.

Our interpretation for the absence of the bundle peaks in the ODMR data is that for neighboring nanotubes the exciton transfer process dominates over the usual relaxation processes. In other words, whenever possible the exciton energy transfer occurs. In case of the $\mathrm{B}_{1}$ line, the exciton is transferred with a probability of 1 from the $(9,4)$ to the $(7,6)$ SWCNT, which does not allow the population of the triplet state, $T$, on the $(9,4)$ nanotube. This explains the insensitivity of the bundlepeak to the ODMR conditions.

The dominance of the Förster exciton energy transfer process was predicted in ref 47 due to the sizable interaction between SWCNTs in a small bundle and the overlapping of optically active states, but to our knowledge the magnitude of its probability has not been quantified yet. The Förster exciton 
energy transfer process is known to play an important role in so-called molecular ruler applications ${ }^{48}$ due to the sensitivity of the process for molecular distances and also for fundamental exciton transfer processes including photosynthesis. ${ }^{49}$ Our work thus allows the role of SWCNTs during applications which involve the exciton energy transfer either from or to SWCNTs to be explored.

\section{CONCLUSIONS}

In conclusion, we studied the optically detected magnetic resonance spectra of SWCNTs by selectively exciting the absorption bands and by analyzing the emitted signal. The study allowed the direct observation of phosphorescence from chirality assigned SWCNTs. The SWCNT diameter dependent singlet-triplet energy gaps were obtained, and they were found to agree well with the value of theoretical predictions; also the expected $1 / d^{2}$ diameter dependence was found. A saturation experiment with varying microwave power allowed the nominal spin-lattice relaxation time of the triplet sublevels to be determined. The bundle-peaks are absent in the ODMR spectra, which is explained by the dominance of the Förster exciton energy transfer between neighboring nanotubes over the usual relaxation processes of excitons. We believe that these findings may enable quantum information storage, manipulation, and readout using telecom compatible wavelengths while using carbon nanotubes.

\section{METHODS}

Single-wall carbon nanotubes were studied in aqueous suspensions following the conventional routes. ${ }^{13,14}$ A solution of sodiumdeoxycholate (DOC) surfactant and distilled water (2\% concentration) was mixed with HiPco nanotubes (Carbon Nanotechnologies Inc.) in an ultrasonic bath. This type of nanotube is known to contain several SWCNT chiralities with a mean diameter of $1 \mathrm{~nm}$ (ref 50).

The nanotube and surfactant mixture was intensively sonicated with a tip-sonicator (Branson Sonifier 450) for $1 \mathrm{~h}$ with $60 \%$ output intensity. During the sonication, the suspension is cooled with a circulating water system. This process makes the nanotubes individualized as the surfactant molecules envelop the nanotubes and help their debundling. The next step is an ultracentrifugation of the samples with $200 \mathrm{~kg}$ acceleration for an hour. During this part of the preparation, the nanotubes, which are not individualized, settle down at the bottom of the cuvette. Finally, the sample is decanted from the top of the suspension and placed into a $4 \mathrm{~mm}$ diameter quartz tube for the measurements.

The development of the ODMR instrument, which allows energy resolved excitation and detection in the near-infrared domain, was reported in ref 19. A tunable laser system, comprising a $532 \mathrm{~nm}$ laser $(5 \mathrm{~W})$ pumped Ti:Sa laser, enables tunable excitation of the SWCNTs, and emitted light is detected in a dispersive monochromator system (Horiba Jobin Yvon ihr320) using a liquid nitrogen cooled InGaAs detector in the $900-1500 \mathrm{~nm}$ range. The samples are in a liquid nitrogen cryostat inside a TE011 microwave cavity which is driven by a TWT amplified microwave signal, whose intensity is chopped. The microwave induced optical signal is measured in phase with the chopped microwaves using a lock-in amplifier, which yields the ODMR signal or $\triangle \mathrm{PL}$. The DC component of the optical signal yields the conventional PL signal, and the two types of signals are measured simultaneously.

\section{ASSOCIATED CONTENT}

\section{s) Supporting Information}

The Supporting Information is available free of charge at https://pubs.acs.org/doi/10.1021/acsnano.0c03139.
Conventional rate equations for the fluorescencephosphorescence system, which is relevant for the ODMR process; additional experimental data to support the identification of the wavelength resolved phosphorescence peaks, namely, excitation profiles; and detailed rate equations for the exciton energy transfer, or the Förster exciton transfer process, which occurs in a small bundle of SWCNTs (PDF)

\section{AUTHOR INFORMATION}

\section{Corresponding Author}

F. Simon - Department of Physics and MTA-BME Lendület Spintronics Research Group (PROSPIN), Budapest University of Technology and Economics, H-1521 Budapest, Hungary; Laboratory of Physics of Complex Matter, Ecole Polytechnique Federale de Lausanne, Lausanne CH-1015, Switzerland; 이이.org/0000-0001-9822-4309; Email: f.simon@ eik.bme.hu

\section{Authors}

J. Palotás - Department of Physics and MTA-BME Lendület Spintronics Research Group (PROSPIN), Budapest University of Technology and Economics, H-1521 Budapest, Hungary; Institute for Molecules and Materials, FELIX Laboratory, Radboud University, 6525ED Nijmegen, The Netherlands

M. Negyedi - Department of Physics and MTA-BME Lendület Spintronics Research Group (PROSPIN), Budapest University of Technology and Economics, H-1521 Budapest, Hungary; Universität Tübingen Physikalische Institut, 72076 Tübingen, Germany; HighFinesse GmbH, 72076 Tübingen, Germany

S. Kollarics - Department of Physics and MTA-BME Lendület Spintronics Research Group (PROSPIN), Budapest University of Technology and Economics, H-1521 Budapest, Hungary

A. Bojtor - Department of Physics and MTA-BME Lendület Spintronics Research Group (PROSPIN), Budapest University of Technology and Economics, H-1521 Budapest, Hungary

P. Rohringer - Faculty of Physics, University of Vienna, Vienna A-1090, Austria

T. Pichler - Faculty of Physics, University of Vienna, Vienna A1090, Austria; O orcid.org/0000-0001-5377-9896

Complete contact information is available at:

https://pubs.acs.org/10.1021/acsnano.0c03139

\section{Author Contributions}

J.P. and M.N. contributed equally to this work. M.N. and F.S. developed and J.P., S.K., and A.B. tested extensively the ODMR spectrometer. M.N. performed the ODMR studies, and J.P. analyzed the data. P.R. and T.P. prepared and characterized the SWCNT samples. F.S. and J.P. developed the kinetic models. All authors contributed to writing the manuscript.

\section{Notes}

The authors declare no competing financial interest.

\section{ACKNOWLEDGMENTS}

Work supported by the Hungarian National Research, Development and Innovation Office (NKFIH) Grant Nos. 2017-1.2.1-NKP-2017-00001 and K119442 and the FP7-GA607491 (COMIQ) and the FWF (P27769-N20) grants. F.S. acknowledges the hospitality of L. Forró during a sabbatical stay and financial support by the Swiss National Science 
Foundation (Grant 200021 144419) and European Research Council (ERC) Advanced Grant No. 670918.

\section{REFERENCES}

(1) Frenkel, J. On the Transformation of Light into Heat in Solids. I. Phys. Rev. 1931, 37, 17.

(2) Kittel, C. Introduction to Solid State Physics, 8th ed.; Wiley: New York, 2004.

(3) Perebeinos, V.; Tersoff, J.; Avouris, P. Scaling of Excitons in Carbon Nanotubes. Phys. Rev. Lett. 2004, 92, 257402.

(4) Spataru, C. D.; Ismail-Beigi, S.; Benedict, L. X.; Louie, S. G. Excitonic Effects and Optical Spectra of Single-Walled Carbon Nanotubes. Phys. Rev. Lett. 2004, 92, 077402.

(5) Wang, F.; Dukovic, G.; Brus, L. E.; Heinz, T. F. The Optical Resonances in Carbon Nanotubes Arise from Excitons. Science 2005, 308, 838-841.

(6) Maultzsch, J.; Pomraenke, R.; Reich, S.; Chang, E.; Prezzi, D.; Ruini, A.; Molinari, E.; Strano, M. S.; Thomsen, C.; Lienau, C. Exciton Binding Energies in Carbon Nanotubes from Two-Photon Photoluminescence. Phys. Rev. B: Condens. Matter Mater. Phys. 2005, 72,241402

(7) Kasha, M. Characterization of Electronic Transitions in Complex Molecules. Discuss. Faraday Soc. 1950, 9, 14-19.

(8) Tsutsui, T.; Yang, M.-J.; Yahiro, M.; Nakamura, K.; Watanabe, T.; Tsuji, T.; Fukuda, Y.; Wakimoto, T.; Miyaguchi, S. High Quantum Efficiency in Organic Light-Emitting Devices with Iridium-Complex as a Triplet Emissive Center. Jpn. J. Appl. Phys. 1999, 38, L1502L1504.

(9) Jelezko, F.; Wrachtrup, J. Single Defect Centres in Diamond: A Review. Phys. Status Solidi A 2006, 203, 3207-3225.

(10) Weber, J. R.; Koehl, W. F.; Varley, J. B.; Janotti, A.; Buckley, B. B.; Van de Walle, C. G.; Awschalom, D. D. Quantum Computing with Defects. Proc. Natl. Acad. Sci. U. S. A. 2010, 107, 8513-8518.

(11) Ciarrocchi, A.; Unuchek, D.; Avsar, A.; Watanabe, K.; Taniguchi, T.; Kis, A. Polarization Switching and Electrical Control of Interlayer Excitons in Two-Dimensional van der Waals Heterostructures. Nat. Photonics 2019, 13, 131-136.

(12) Unuchek, D.; Ciarrocchi, A.; Avsar, A.; Watanabe, K.; Taniguchi, T.; Kis, A. Room-Temperature Electrical Control of Exciton Flux in a van der Waals Heterostructure. Nature 2018, 560, $340-344$.

(13) O'Connell, M. J.; Bachilo, S. M.; Huffman, C. B.; Moore, V. C.; Strano, M. S.; Haroz, E. H.; Rialon, K. L.; Boul, P. J.; Noon, W. H.; Kittrell, C.; Ma, J. P.; Hauge, R. H.; Weisman, R. B.; Smalley, R. E. Band Gap Fluorescence from Individual Single-Walled Carbon Nanotubes. Science 2002, 297, 593.

(14) Bachilo, S. M.; Strano, M. S.; Kittrell, C.; Hauge, R. H.; Smalley, R. E.; Weisman, R. B. Structure-Assigned Optical Spectra of Single-Walled Carbon Nanotubes. Science 2002, 298, 2361-2366.

(15) Mortimer, I. B.; Nicholas, R. J. Role of Bright and Dark Excitons in the Temperature-Dependent Photoluminescence of Carbon Nanotubes. Phys. Rev. Lett. 2007, 98, 027404.

(16) Lebedkin, S.; Hennrich, F.; Kiowski, O.; Kappes, M. M. Photophysics of Carbon Nanotubes in Organic Polymer-Toluene Dispersions: Emission and Excitation Satellites and Relaxation Pathways. Phys. Rev. B: Condens. Matter Mater. Phys. 2008, 77, 165429.

(17) Crochet, J.; Clemens, M.; Hertel, T. Quantum Yield Heterogeneities of Aqueous Single-Wall Carbon Nanotube Suspensions. J. Am. Chem. Soc. 2007, 129, 8058-8059.

(18) Kim, Y.; Goupalov, S. V.; Weight, B. M.; Gifford, B. J.; He, X.; Saha, A.; Kim, M.; Ao, G.; Wang, Y.; Zheng, M.; Tretiak, S.; Doorn, S. K.; Htoon, H. Hidden Fine Structure of Quantum Defects Revealed by Single Carbon Nanotube Magneto-Photoluminescence. ACS Nano 2020, 14, 3451-3460.

(19) Negyedi, M.; Palotás, J.; Gyüre, B.; Dzsaber, S.; Kollarics, S.; Rohringer, P.; Pichler, T.; Simon, F. An Optically Detected Magnetic Resonance Spectrometer with Tunable Laser Excitation and Wave- length Resolved Infrared Detection. Rev. Sci. Instrum. 2017, 88, 013902.

(20) Capaz, R. B.; Spataru, C. D.; Ismail-Beigi, S.; Louie, S. G. Excitons in Carbon Nanotubes: Diameter and Chirality Trends. Phys. Status Solidi B 2007, 244, 4016-4020.

(21) Giacometti, G.; Agostini, G.; Santabarbara, S.; Carbonera, D. ODMR Spectroscopy of Molecular Functions in Photosynthetic Membrane Proteins. Appl. Magn. Reson. 2007, 31, 179.

(22) Carbonera, D. Optically Detected Magnetic Resonance (ODMR) of Photoexcited Triplet States. Photosynth. Res. 2009, 102, 403.

(23) Kwiram, A. L.; Ross, J. B. Optical Detection of Magnetic Resonance in Biologically Important Molecules. Annu. Rev. Biophys. Bioeng. 1982, 11, 223.

(24) Clarke, R. H. Triplet State ODMR Spectroscopy: Techniques and Applications to Biophysical Systems; Wiley: New York, 1982.

(25) Wang, F.; Dukovic, G.; Brus, L. E.; Heinz, T. F. Time-Resolved Fluorescence of Carbon Nanotubes and Its Implication for Radiative Lifetimes. Phys. Rev. Lett. 2004, 92, 177401.

(26) Misewich, J.; Martel, R.; Avouris, P.; Tsang, J.; Heinze, S.; Tersoff, J. Electrically Induced Optical Emission from a Carbon Nanotube FET. Science 2003, 300, 783-786.

(27) Gabor, N. M.; Zhong, Z.; Bosnick, K.; Park, J.; McEuen, P. L. Extremely Efficient Multiple Electron-Hole Pair Generation in Carbon Nanotube Photodiodes. Science 2009, 325, 1367-1371.

(28) Childress, L.; Gurudev Dutt, M. V.; Taylor, J. M.; Zibrov, A. S.; Jelezko, F.; Wrachtrup, J.; Hemmer, P. R.; Lukin, M. D. Coherent Dynamics of Coupled Electron and Nuclear Spin Qubits in Diamond. Science 2006, 314, 281-285.

(29) Neumann, P.; Kolesov, R.; Naydenov, B.; Beck, J.; Rempp, F.; Steiner, M.; Jacques, V.; Balasubramanian, G.; Markham, M. L.; Twitchen, D. J.; Pezzagna, S.; Meijer, J.; Twamley, J.; Jelezko, F.; Wrachtrup, J. Quantum Register Based on Coupled Electron Spins in a Room-Temperature Solid. Nat. Phys. 2010, 6, 249-253.

(30) Nagatsu, K.; Chiashi, S.; Konabe, S.; Homma, Y. Brightening of Triplet Dark Excitons by Atomic Hydrogen Adsorption in SingleWalled Carbon Nanotubes Observed by Photoluminescence Spectroscopy. Phys. Rev. Lett. 2010, 105, 157403.

(31) Stich, D.; Späth, F.; Kraus, H.; Sperlich, A.; Dyakonov, V.; Hertel, T. Triplet-Triplet Exciton Dynamics in Single-Walled Carbon Nanotubes. Nat. Photonics 2014, 8, 139-144.

(32) Ishii, A.; Machiya, H.; Kato, Y. K. High Efficiency Dark-ToBright Exciton Conversion in Carbon Nanotubes. Phys. Rev. X 2019, 9, 041048.

(33) Crochet, J. J.; Sau, J. D.; Duque, J. G.; Doorn, S. K.; Cohen, M. L. Electrodynamic and Excitonic Intertube Interactions in Semiconducting Carbon Nanotube Aggregates. ACS Nano 2011, 5, 26112618.

(34) Weisman, R. B.; Bachilo, S. M. Dependence of Optical Transition Energies on Structure for Single-Walled Carbon Nanotubes in Aqueous Suspension: An Empirical Kataura Plot. Nano Lett. 2003, 3, 1235-1238.

(35) Torrens, O. N.; Milkie, D. E.; Zheng, M.; Kikkawa, J. M. Photoluminescence from Intertube Carrier Migration in SingleWalled Carbon Nanotube Bundles. Nano Lett. 2006, 6, 2864-2867.

(36) Tan, P. H.; Rozhin, A. G.; Hasan, T.; Hu, P.; Scardaci, V.; Milne, W. I.; Ferrari, A. C. Photoluminescence Spectroscopy of Carbon Nanotube Bundles: Evidence for Exciton Energy Transfer. Phys. Rev. Lett. 2007, 99, 137402.

(37) Htoon, H.; O'Connell, M. J.; Cox, P. J.; Doorn, S. K.; Klimov, V. I. Low Temperature Emission Spectra of Individual Single-Walled Carbon Nanotubes: Multiplicity of Subspecies within Single-Species Nanotube Ensembles. Phys. Rev. Lett. 2004, 93, 027401.

(38) Capaz, R. B.; Spataru, C. D.; Ismail-Beigi, S.; Louie, S. G. Diameter and Chirality Dependence of Exciton Properties in Carbon Nanotubes. Phys. Rev. B: Condens. Matter Mater. Phys. 2006, 74, 121401.

(39) Piao, Y.; Meany, B.; Powell, L. R.; Valley, N.; Kwon, H.; Schatz, G. C.; Wang, Y. Brightening of Carbon Nanotube Photoluminescence 
through the Incorporation of sp3 Defects. Nat. Chem. 2013, 5, 840845.

(40) Slichter, C. P.; Principles of Magnetic Resonance, 3rd ed.; Spinger-Verlag: New York, 1989.

(41) Poole, C. P.; Electron Spin Resonance, 2nd ed.; John Wiley \& Sons: New York, 1983.

(42) Portis, A. M. Electronic Structure of F Centers: Saturation of the Electron Spin Resonance. Phys. Rev. 1953, 91, 1071.

(43) Žutić, I.; Fabian, J.; Das Sarma, S. Spintronics: Fundamentals and Applications. Rev. Mod. Phys. 2004, 76, 323-410.

(44) Abragam, A. Principles of Nuclear Magnetism; Oxford University Press: Oxford, U.K., 1961.

(45) Yoshikawa, K.; Matsunaga, R.; Matsuda, K.; Kanemitsu, Y. Mechanism of Exciton Dephasing in a Single Carbon Nanotube Studied by Photoluminescence Spectroscopy. Appl. Phys. Lett. 2009, 94, 093109.

(46) Jarmola, A.; Acosta, V. M.; Jensen, K.; Chemerisov, S.; Budker, D. Temperature- and Magnetic-Field-Dependent Longitudinal Spin Relaxation in Nitrogen-Vacancy Ensembles in Diamond. Phys. Rev. Lett. 2012, 108, 197601.

(47) Lefebvre, J.; Finnie, P. Photoluminescence and Förster Resonance Energy Transfer in Elemental Bundles of Single-Walled Carbon Nanotubes. J. Phys. Chem. C 2009, 113, 7536-7540.

(48) Tskhovrebova, L.; Trinick, J. Molecular Rulers? Curr. Biol. 2012, 22, R317-R318.

(49) Förster, T. Delocalized Excitation and Excitation Transfer; Florida State University: Tallahassee, FL, U.S.A., 1965.

(50) Kukovecz, A.; Kramberger, C.; Georgakilas, V.; Prato, M.; Kuzmany, H. A Detailed Raman Study on Thin Single-Wall Carbon Nanotubes Prepared by the HiPCO Process. Eur. Phys. J. B 2002, 28, 223-230. 